\section{An automated continuous monitoring system: a useful tool for monitoring neuronal differentiation of human embryonic stem cells}

\author{
Tuomas Tapani Huttunen, ${ }^{1}$ \\ Maria Sundberg, ${ }^{1}$ Harri Pihlajamäki, ${ }^{2}$ \\ Riitta Suuronen, ${ }^{1,3,4}$ Heli Skottman, ${ }^{1}$ \\ Riikka Äänismaa, ${ }^{1}$ Susanna Narkilahti' ${ }^{1}$ \\ ${ }^{1}$ Regea - Institute for Regenerative \\ Medicine, University of Tampere and \\ Tampere University Hospital, Tampere; \\ ${ }^{2}$ Centre for Military Medicine, Helsinki; \\ ${ }^{3}$ Department of Eye, Ear and Oral \\ Diseases, Tampere University Hospital, \\ Tampere; ${ }^{4}$ Department of Biomedical \\ Engineering, Tampere University \\ of Technology, Tampere, Finland
}

\section{Abstract}

The currently used cell culturing and differentiation procedures are both time- and laborintensive. Automation of some of these procedures will increase the efficiency of commonly used cell differentiation protocols. We used a particular cell culture platform to rapidly and efficiently screen the neuronal differentiation of human embryonic stem cells (hESC). Continuous live monitoring and analysis of non-labeled cells using this system allowed us to characterize neuronal populations over the entire neuronal differentiation process. The differentiation of individual cells from early progenitor cells to neurons and glial cells could be monitored continuously using this system with sub-confluent cell cultures. The imaged data was collected and analyzed with a specially designed cell recognition protocol, which resulted in a quantitative neuronal cell count. The analysis results were confirmed using conventional laboratory methods such as manual counting and flow cytometry. Our findings suggest that an automated culture platform combined with automated monitoring and analysis systems is a reliable method for developing enhanced cell differentiation procedures or as part of an automated quality control system for existing protocols.

\section{Introduction}

Pure neuronal populations derived from human embryonic stem cells (hESC) are a potentially ideal source material in the field of regenerative medicine. ${ }^{1}$ Microscopic evalua- tion, gene expression analysis, immunocytochemical characterization, and electrophysiologic recordings are commonly used to obtain data regarding cell division, maturation, fiber growth, and death of hESC-derived neuronal cells. $^{2-5}$ These conventional methods, however, are labor intensive and do not provide information about cellular movement, interactions, or behavior of the neuronal cells in vitro.

More sophisticated systems are needed for the efficient culturing and evaluation of hESCs and hESC-derived neuronal cells to enable both optimization of differentiation protocols and the development of automated quality control systems. Automated culturing systems for hESC cultures were recently developed. ${ }^{6,7}$ Visual evaluation and photography of cell cultures and cells, however, are also time-consuming. Time-lapse imaging can be used to obtain sequential images of neuronal cells that can be converted into a movie format for the evaluation of cellular events in neuronal populations. ${ }^{8}$ Similar systems have been described with mouse ESCs. ${ }^{9,10}$ Moreover, the use of time-lapse imaging allows for semi-automated identification of axonal growth cones. ${ }^{11}$ The development of a system that combines automated culture with automated image capture and analysis would greatly facilitate the efficient screening of hESC-derived neuronal cells for therapeutic purposes.

We previously demonstrated that an automated continuous monitoring system is useful for surveillance of hESC cultures. ${ }^{12}$ Here, we extend the use of this system for monitoring, characterizing, and analyzing hESC-derived neuronal cells. The system proved to be a useful tool for optimizing hESC-derived neuronal cell differentiation protocols, thereby increasing the speed and efficiency of hESC screening.

\section{Materials and Methods}

\section{Cell culture}

\section{hESC culture}

The hESC lines HS181, HS293, and HS360, derived at the Fertility Unit of Karolinska University Hospital Huddinge, Karolinska Institute, Sweden, were cultured on a feeder cell layer of either irradiated or mitomycin Ctreated commercially available human fibroblasts (American Type Culture Collection, Manassas, VA, USA; http://www.lgcpromochem-atcc.com), as described previously. ${ }^{13-}$ ${ }^{15}$ The Karolinska Institute has the approval of the Ethics Committee of the Karolinska Institute to derive, characterize, and differentiate hESC lines. Regea - Institute for Regenerative Medicine, University of Tampere, Finland, was approved by the Ethics
Correspondence: Susanna Narkilahti, Regea, Institute for Regenerative Medicine, University of Tampere, Biokatu 12 6th floor, 33520 Tampere, Finland.

Tel. +358.40.7085113 - Fax: +358.3.35518498.

E-mail: susanna.narkilahti@uta.fi

Key words: human embryonic stem cells, neura differentiation, automated imaging, machine vision.

Acknowledgements: Professor Hovatta is acknowledged for providing hESC lines HS181, HS293, and HS360 during years 2005-2008. We thank the personnel of Regea for their support in stem cell research. Dr. Antti Lehmussola, Tampere University of Technology, is acknowl edged for technical advice and MSc Johanna Ketolainen for flow cytometric analysis. The study was funded by the Academy of Finland; the Competitive Research Funding of Pirkanmaa Hospital District; the Employment and Economic Development Center for Pirkanmaa; the Finnish Defense Forces; and TEKES, the Finnish Funding Agency for Technology and Innovation.

Contributions: TTH, AR, design and implementation of the study, interpretation of the results, and writing of the manuscript; MS, implementation of FACS analysis; HP, RS, HS, design of the study; $\mathrm{SN}$, design and supervision of the study, writing and final approval of the manuscript.

Conflict of interests: the authors report no conflicts of interest.

Received for publication: 27 May 2011

Accepted for publication: 18 July 2011.

This work is licensed under a Creative Commons Attribution NonCommercial 3.0 License (CC BYNC 3.0).

(C) Copyright T.T. Huttunen et al., 2011

Licensee PAGEPress, Italy

Stem Cell Studies 2011; 1:e10

doi:10.4081/scs.2011.e10

Committee of Pirkanmaa Hospital District to culture hESC lines derived at the Karolinska Institute. The hESC culture medium comprised Knockout DMEM (Invitrogen, Carlsbad, CA, USA; http://www.invitrogen.com), 20\% Serum Replacement (Invitrogen), $2 \mathrm{mM}$ GlutaMax (Invitrogen), 1\% non-essential amino acids (Cambrex Bio Science, East Rutherford, NJ, USA; http://www.cambrex. com), $50 \mathrm{U} / \mathrm{mL}$ penicillin/streptomycin (Cambrex Bio Science Inc), $0.1 \mathrm{mM}$ 2-mercaptoethanol (Invitrogen), and $8 \mathrm{ng} / \mathrm{mL}$ basic fibroblast growth factor (bFGF, R\&D Systems, Minneapolis, MN, USA; http://www.rndsystems.com). Colonies were mechanically passaged on a weekly basis. The undifferentiated state of the colonies was confirmed daily by visual morphologic analysis and periodic immunocytochemical testing for the expres- 
sion of the embryonic stem cell markers Nanog, OCT-4, SSEA-4, and Tra-1-60. Karyotype testing was performed frequently, indicating that all hESC lines used in this study had normal karyotypes.

\section{Differentiation of neuronal cells}

Neuronal differentiation was initiated after mechanically splitting non-differentiated hESC colonies (HS181 p60-75, HS293 p50-63, and HS360 p45-57) into aggregates containing a few hundred cells. ${ }^{16,17}$ Prior to mechanical splitting, hESC colonies were cultured in neuronal induction medium overnight. The neuronal induction medium consisted of $1: 1$ of DMEM/F12 (Invitrogen) and Neurobasal (Invitrogen), $2 \mathrm{mM}$ GlutaMax, 1× B27 (Invitrogen), 1× N2 (Invitrogen), $25 \mathrm{U} / \mathrm{mL}$ penicillin/streptomycin, and $20 \mathrm{ng} / \mathrm{ml} \mathrm{bFGF}$. The small hESC aggregates were grown on uncoated cell culture dishes (CellBIND Surface, Corning Inc., Corning, NY, USA; http://www.corning.com). The aggregates attached to the wells 2 to $3 \mathrm{~d}$ after seeding and started growing as monolayers. Adherent colonies began to form typical neuronal rosette-like structures 7 to $14 \mathrm{~d}$ after initiating neuronal induction, as described earlier. ${ }^{4,18}$ The rosette-like structures were mechanically dissected using surgical scalpels and replated on poly-l-lysine $(0.1 \mathrm{mg} / \mathrm{mL}$, Sigma-Aldrich, St. Louis, MO, USA; http://www.sigmaaldrich.com)/laminin (10 $\mu \mathrm{g} / \mathrm{mL}$, Sigma) - coated cell culture dishes (Nunclon surface, Nunc Inc., Roskilde, Denmark; http://www. nuncbrand.com) in the absence of bFGF. The replated and passaged rosettes began forming adherent centers (rosette centers) that produced neuronal progenitor cells. Periodically, adherent rosette centers were dissected mechanically and replated onto new poly-l-lysine/laminin-coated dishes. After replating, bFGF was routinely omitted from the neuronal media to enhance neuronal differentiation. For longer culture periods (7-21 days), bFGF ( $4 \mathrm{ng} / \mathrm{mL}$ ) was added to the media with or without brain-derived neurotrophic factor (BDNF, $5 \mathrm{ng} / \mathrm{mL}$; Invitrogen). All the following experiments were performed with these monolayer cultures.

\section{Online monitoring of neuronal differentiation with the automated culture platform}

\section{Instrumentation}

A detailed description of the Cell-IQ ${ }^{\circledR}$ (ChipMan Technologies, Tampere, Finland; http://www.chipmantech.com) cell culture platform instrumentation was published previously. ${ }^{12}$ This system comprises a controlled culture environment, a phase-contrast microscope $(10 \times$ objective thus $100 \times$ magnifica- tion), and a camera that are integrated into an automated cell monitoring and analysis system. The system utilizes machine vision technology, which has traditionally been used in the fields of medical imaging, precision robotics, and object recognition, and is one of the first of its kind designed for studying biologic processes. ${ }^{19,20}$ In the controlled environment, the temperature $\left(36.5^{\circ} \mathrm{C}\right)$, humidity, and $5 \%$ $\mathrm{CO}_{2}$ atmosphere (piped directly onto the cells) mimics the conditions in a typical cell incubator. Cells grown on culture plates (2 to 92 - well plates) are placed on an integrated plate holder that enables controlled xy $( \pm 1 \mu \mathrm{m})$ movement of the plates. An automated optics module containing phase-contrast optics, a CCD (charge-coupled device) camera, and a green LED light are controlled by machine visionbased firmware. The motorized z stage $( \pm 0.4$ $\mu \mathrm{m})$ utilizes a dynamic Z-stack (user defined) that creates all-in-focus images $(506 \times 675 \mu \mathrm{m})$ from the regions of interest. ${ }^{21}$ For monitoring larger areas, single all-in-focus images can be combined into stitched grid images $(1 \times 1$ to $12 \times 12)$. Single captured images are stored in separate folders in a JPEG-format that can be opened and transferred into a movie format for post-hoc analysis of the behavior of single cells or cell colonies. The analysis software can be used to build user-defined cell recognition programs that enable the rapid analysis of cell types, neurite outgrowth, cell division, and other events from captured images. A schematic figure of the cell culture and monitoring process is shown in Figure 1.

\section{Monitoring the differentiating neural culture}

After hESC-derived neural cells were allowed to attach for 1 to 2 days in the cell incubator, 12-well plates were placed on the Cell-IQ culturing platform. Cultures were monitored during the entire hESC neuronal differentiation process as described above. The represen- tative areas or cells of interest were selected from the well plates. These locations were marked in $1 \times 1$ to $6 \times 6$ grid positions to a well plate control map that stored the positions as $\mathrm{x}-, \mathrm{y}-, \mathrm{z}$-coordinates. Marked positions were imaged in pre-defined time-lapse cycles for several days to weeks. The saved cycle files containing the position coordinates allowed to stop the imaging, removing the well plate from Cell-IQ, changing of the culture media (every second or third day), and reloading the same cycle file for the same well plate. Thus, the areas of interest could be imaged for indefinite amount of time.

The neuronal cell and neurite analysis protocol

The neuronal cell analysis protocol was created using Cell-IQ Analyser software. For the neuronal cell recognition protocol, cells were first imaged the with Cell-IQ system and then immediately fixed and immunostained with neuronal cell markers (see Immunocytochemistry and Microscopy below for details). The last captured Cell-IQ images were compared with the immunocytochemical staining to recognize neuronal cells and flat epithelial-like cells from the other cells in the colonies (Figure 2).

These data were used to classify cells into separate categories. For the neuronal cell recognition protocol, individual cells of different types were categorized into following defined groups: i) neuronal cells, ii) flat epithelial-like cells and glial cells, as well as: iii) dense colony area, iv) white dot, and v) cell debris. The main focus of the build protocol was the recognition of individual neurons from the other cells. Representative samples for each category ( 100 samples/category) were collected from various images. A typical sample collection from a single image is shown in Figure 3. The collected sample library was sent to the manufacturer (Chip-Man Technologies) where the neuronal cell recog-
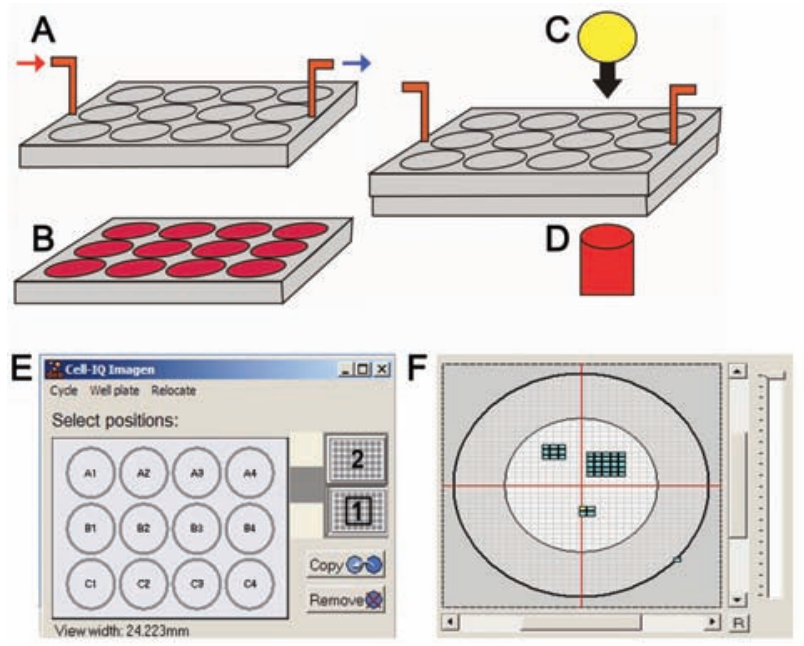

Figure 1. Automated monitoring of differentiating cells. A specially designed lid (A) with an in- and outflow channel for gas is placed tightly on top of a regular 12well cell culture plate (B). A LED light (C) illuminates the wells for the camera (D) to automatically image the selected positions. Graphical well-plate control $(E, F)$ is used to select positions on wells for monitoring. The desired plate and positions are selected $(\mathrm{E})$, as well as the grid size (F). 
nition protocol was built and tested for correct cell recognition. We then tested the neuronal cell recognition protocol with a small series of images and a few corrections were made to the sample collection to improve the recognition accuracy.

\section{Immunocytochemistry and microscopy}

After monitoring the cells, the cells were fixed with $4 \%$ paraformaldehyde for $20 \mathrm{~min}$ at room temperature and washed twice with phosphate buffered saline (PBS). The fixed cells were blocked for 45 min using 10\% normal donkey serum (NDS), 0.1\% Triton X-100, and $1 \%$ bovine serum albumin (BSA) in PBS. The blocked samples were washed once with $1 \%$ NDS, $0.1 \%$ Triton X-100, and 1\% BSA in PBS and then incubated overnight at $+4^{\circ} \mathrm{C}$ with primary antibodies in 1\% NDS, $0.1 \%$ Triton X-100, and $1 \%$ BSA in PBS. Primary antibodies used for single- and double- labeling were: antiA2B5 (1:600), anti- $\beta$-tubulin ${ }_{\text {III }}$ (1:1000), and anti-neurofilament (NF) -200 (1:600) purchased from Sigma; monoclonal rabbit antimicrotubule-associated protein (MAP-2; 1:400), monoclonal mouse anti-nestin (1:1000), monoclonal mouse anti-polysialic acid-neural cell adhesion molecule (PSANCAM, 1:500), and polyclonal goat antivimentin (1:200) purchased from Chemicon (Chemicon International Inc., Temecula, CA, USA); sheep anti-glial fibrillary acidic protein (GFAP, 1:400) purchased from R\&D Systems; monoclonal mouse anti-endoglin (CD105; 1:200), and anti- growth associated protein (GAP-43; 1:200) purchased from Santa Cruz Biotechnology Inc. (Santa Cruz, CA, USA).

After incubation, the samples were washed three times in 1\% BSA in PBS. The secondary antibodies in 1\% BSA in PBS were then applied for $1 \mathrm{~h}$ at room temperature. The secondary antibodies, either Rhodamine Red (Jackson ImmunoResearch Laboratories Inc., West Grove, PA, USA) or Alexa Fluor-488 (Invitrogen), were conjugated to mouse, goat, sheep, or rabbit antibodies. The samples were then washed three times with PBS and twice with phosphate buffer prior to drying and mounting with Vectashield (Vector Laboratories Inc., Burlingame, CA, USA). Specificity was tested by omitting the primary antibody from the immunocytochemical protocol, which resulted in the disappearance of all positive staining.

Microscopic analysis was performed using an inverted microscope (Eclipse TE2000-S, Nikon, Tokyo, Japan) and pictures were taken with a digital camera (CO0LPIX5400, Nikon, Tokyo, Japan) or an Olympus microscope (IX51S8F-2, Olympus, Tokyo, Japan) equipped with a fluorescence unit and a camera (DP30BW). Adobe Photoshop ${ }^{\circledR}$ (version 9.0 Adobe Systems Inc., San Jose, CA, USA) was used to create the overlaid images.

\section{Flow cytometry}

For flow cytometric analysis, subsets of neuronal cultures monitored with Cell-IQ were labeled with CD105 or MAP-2. Briefly, hESCderived neural cells (HS181 and HS360) were trypsinized for $10 \mathrm{~min}$ at $37^{\circ} \mathrm{C}$. Trypsin-EDTA (BioWhittaker, Fisher Scientific Inc., Leicestershire, UK) was inactivated using 5\% human serum (HS) in PBS. Cells were dissociated into single-cell suspension by trituration, centrifuged $1500 \mathrm{rpm}, 5 \mathrm{~min}+4^{\circ} \mathrm{C}$ and resuspended in PBS containing 5\% HS. Cells were counted and filtered thereafter using $50 \mu \mathrm{m}$ cell-strainers (CupFilcons, BD Biosciences, Franklin Lakes, NJ, USA). For CD105 analysis, aliquots of 100000 viable cells per sample were directly labeled with anti-human CD105-PE
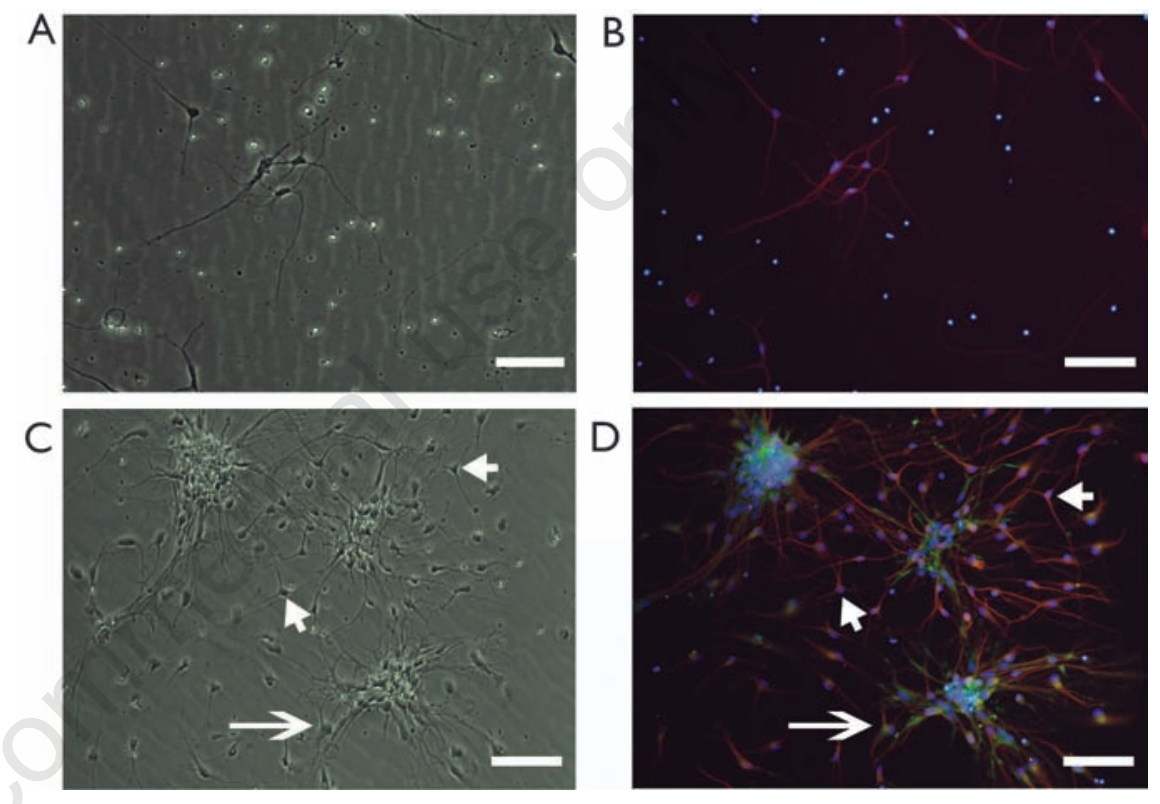

Figure 2. Characterization of differentiating neuronal cells. (A) Cells with neuronal morphology and well-defined soma and fibers were $\beta$-tubulin ${ }_{\text {III-positive neurons (red) from }}$ the corresponding area in (B). (C) Cells with loose, undefined morphology (long white arrow), in contrast to neuronal-like cells (short white arrows), were (D) labeled with the glial marker, vimentin (green, long arrow). DAPI (blue). Scale bars: A-D = $100 \mu \mathrm{m}$.
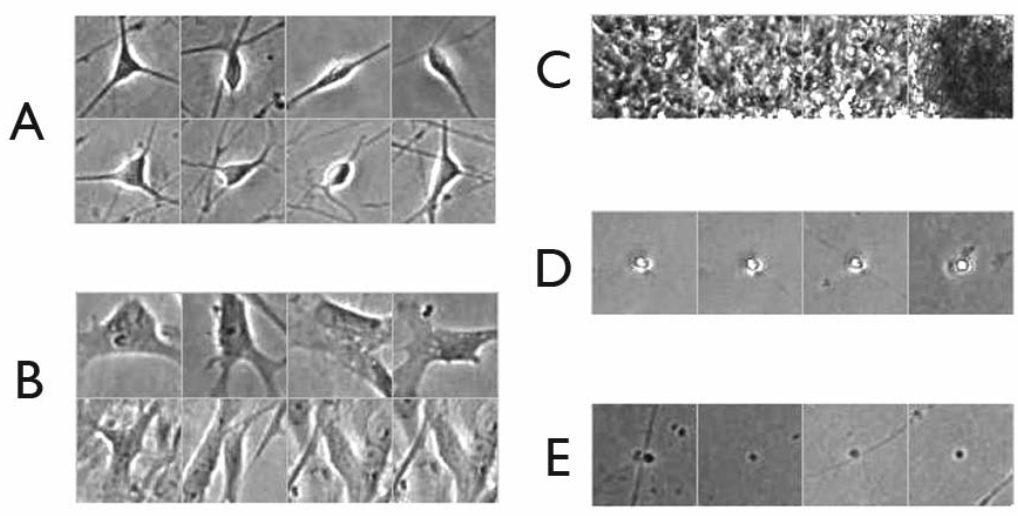

Figure 3. Cell types for the recognition process. For this experiment, the recognition software was taught to identify 5 object types. Neurons (A), flat epithelial-like cells (B), colonies (C), small white cells (D), and debris (E). 
was excluded using an unstained control cell sample (fixed cells that did not undergo antibody treatment) and with a non-specifically stained control-sample (primary antibody omitted from the staining protocol). The cell population of interest was determined and dead cells were excluded using forward and side scatter parameters. For each sample, 20,000 events were recorded and analyzed.

\section{Results}

\section{Monitoring of neuronal differentia-} tion in the automated culture system

One to three days after neuronal induction, the culture plates were transferred into the automated cell culture platform where the

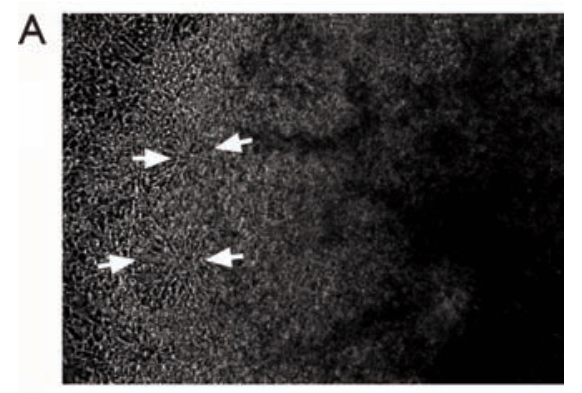

B

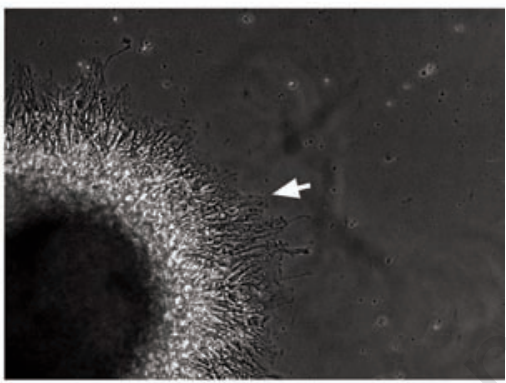

C

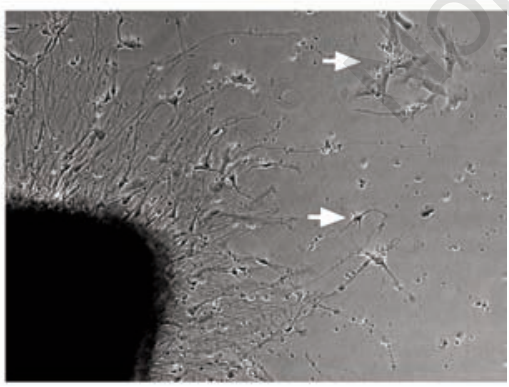

Figure 4. Differentiating neuronal cells grown on an automated cell culture and monitoring platform. (A) A colony with the rosette formations (white arrows) 10 days after the initiation of differentiation. (B) Rosette with a dense outgrowth of fiber-like processes (white arrow) mechanically separated and replated on new laminin-coated dish. (C) Replated neuronal differentiating colony with flat epithelial-like cells (arrows). Images taken with Cell-IQ platform Scale bars $=200 \mu \mathrm{m}$. growth of differentiating colonies was monitored $24 \mathrm{~h} / \mathrm{d}$. All of the grayscale figures and movies presented here are data obtained using the automated imaging system. In the center of the colonies, visible neuronal rosettes formed after 7 to $14 \mathrm{~d}$ in induction medium (Figure 4A), as described previously. ${ }^{4}$ All the colonies originating from the HS181 and HS360 lines produced at least one neuronal rosette area, whereas only $50 \%$ of colonies from the HS293 line produced neuronal rosettes. Mechanically dissected rosettes attached within $1 \mathrm{~d}$, after which the plates were transferred back into the automated culture system for continuous monitoring. Fiberlike processes grew rapidly out from the rosette centers and were easily detected in the all-in-focus images (Figure 4B). At the same time, flat epithelial-like cells were observed (Figure 4C). Mechanical dissection and replating of the rosette centers gave rise to purer neuronal cultures (neuronal centers) from which PSA-NCAM positive fiber outgrowth were detected. This was followed by a radial
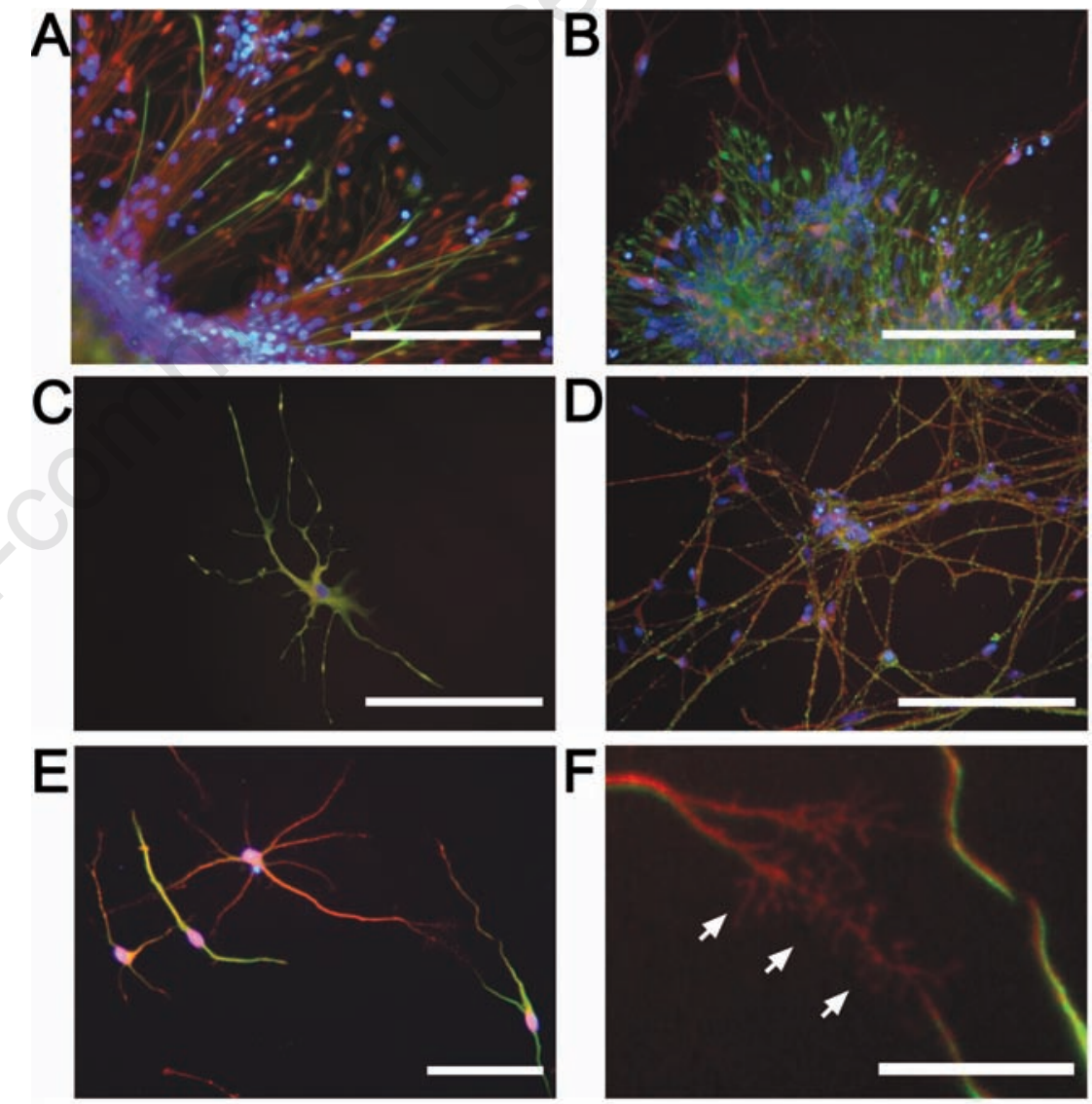

Figure 5. Characterization of differentiating neuronal cells. (A) Mechanically dissected early neuronal rosettes had nestin- (red) and GFAP- (green) positive fibers. (B) Outgrowth

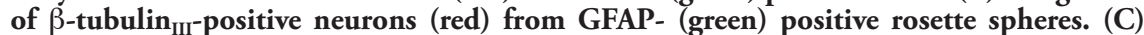
Mature GFAP- (green) and nestin- (red) positive astrocyte. (D) $\beta$-tubulin III- (red) positive neuronal networks positive for GAP-43 (green). (E) MAP-2 (green) and NF-200 (red) localize in different compartments of the same neuron. (F) Detailed picture of an area from Figure $5 \mathrm{E}$ showing a hairy synaptic contact (white arrows) between two neurons. DAPI staining as blue in A-E. Scale bars: A-E=50 $\mu \mathrm{m}, \mathrm{F}=\mathbf{2 5} \mu \mathrm{m}$. 
5B). For 2 to 3 wk, neuronal maturation continued with low concentrations of bFGF and BDNF in the medium. After $4 \mathrm{wk}$, there were nestinand GFAP-positive glial cells with a mature astrocytic morphology in the cultures (Figure 5C). In parallel, $\beta$-tubulin III $_{\text {-positive neurons }}$ grew out from the neuronal spheres and formed loose fiber networks that were positive for GAP-43 (Figure 5D). After 4 to $5 \mathrm{wk}$, there was MAP-2- (associated with dendrites in adult neurons) and NF-200- (associated with axons in adult neurons) positive labeling in different subcellular compartments of the same neurons (Figure 5E). In addition, we detected synaptic contacts with MAP-2- and NF-200-positive hairy fibers between the neurons (Figure 5F). The flat epithelial-like cells were found to be positive for CD105 i.e. endoglin with flow cytometric analysis (data not shown) and with immunostaining (Supplemental Figure 1).

\section{Development and validation of neuronal cell recognition protocol}

Recognition of neuronal cells from Cell-IQ images

We compared the morphologic data obtained using the automated monitoring system to that obtained with the immunocytochemical staining. MAP-2 (Figure 6B), and $\beta$-tubulin ${ }_{\text {III }}$ labeling (Figure 2B,D) of cells that had a neuronlike morphology in the last captured image (Figure 6A and Figure 2A,C, respectively) confirmed that these cells were neurons. Neuronal characteristics of cells were further confirmed with double-labeling with vimentin (Figure 2D) and GFAP (data not shown) revealing two distinct populations with different morphologies (Figure 2D, Supplemental online video 3 ).

\section{Neuronal cell analysis}

The neuronal cell recognition program was developed as described above (see Materials and Methods section). The image data analysis using the neuronal cell recognition protocol allowed us to calculate the total number of a cell type/image and the total cell number/ image. In addition, the analysis method automatically counted the neurite length/single image in pixels ( 1 pixel $=0.879 \mu \mathrm{m}$, representation of neurite analysis in Supplemental Figure 2). Cell-IQ analysis software automatically presented the data in a curve graft where the number of objects in different categories (y-axis) is presented against the time (x-axis) as observed in Figure 7D. These data were easily imported into spreadsheets for further analysis.

Validation of the built neuronal cell recognition program

We then compared the results of the automated recognition protocol with two other methods. Briefly, neuronal cell cultures in four 12-well plates were monitored with Cell-IQ (600 grid images), after which half of the cells were fixed and stained with MAP-2 antibody. All together, the portion of MAP-2 positive cells from all cells (DAPI positive nuclei, $\mathrm{n} \sim 8300$ ) was counted manually from 24 wells (12 visual fields/well with $10 \times$ optical magnification). The cells in the remaining 24 wells were suspended and stained with MAP-2 antibody and analyzed using FACS (8 parallel samples). The comparison revealed that in the ideal situation, the Cell-IQ neuronal recognition protocol vs. manual MAP-2 counting vs. MAP-2 FACS analysis identified $55 \%$ vs. $50 \%$ vs. $50 \%$ of the cells in the culture as being neuronal cells (Figure 7). If the neuronal cell colony grew confluent, however, the Cell-IQ neuronal recognition protocol designed to recognize individual neurons was unable to count the neuronal cells reliably as the cells grew too tightly next to each other. Here, the Cell-IQ neuronal cell count was lower compared to the manually or FACS analyzed MAP-2 positive cells: $28 \%$ vs. $45 \%$ vs. $43 \%$ for HS181-derived cultures, and $33 \%$ vs. $83 \%$ vs. $83 \%$ for HS360derived cultures, respectively (data not shown). To conclude, 100000 proliferating neuronal cells $/ \mathrm{cm}^{2}$ plated on 24-well plate can be imaged and analyzed reliably for approximately 7 days. To verify the validation of the system we also differentiated hESCs to neural cells for up to 12 weeks as described earlier, ${ }^{17,22}$
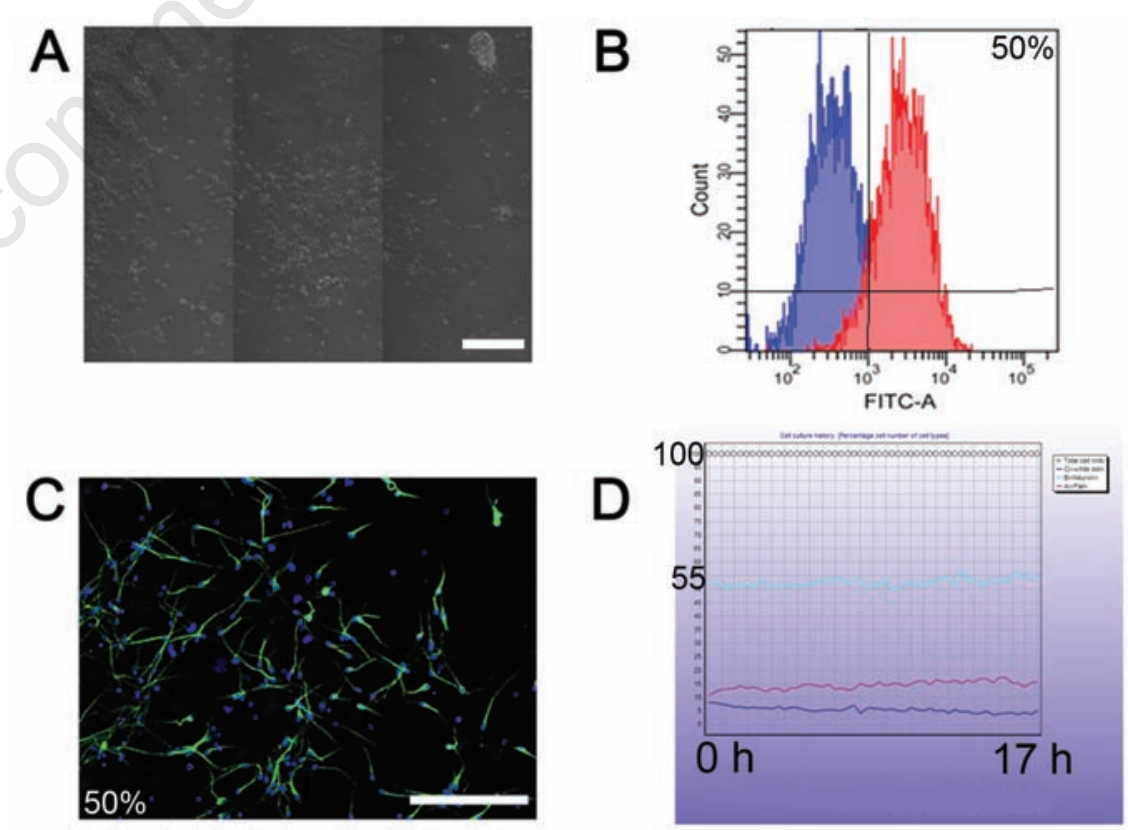

Figure 7. Comparing the results of FACS and immunocytochemistry to Cell-IQ. Similar results were obtained when using Cell-IQ image based analysis (A), MAP-2 positive cells using FACS (B), and manual counting using MAP-2 dye (C). The corresponding analysis results are shown in (D) where Cell-IQ analysis software automatically present the data in a curve graft where the number of objects in different categories (y-axis) is presented against the time ( $x$-axis). After a 9-week differentiation period, MAP-2 positive cells using FACS were at $50 \%$, manual counting using MAP-2 dye gave $50 \%$, and automated analysis indicated approximately $55 \%$ for a single well. Scale bar $A=300 \mu \mathrm{m}$ and $C=200 \mu \mathrm{m}$. 
cell counting was performed as described above. Also this analysis showed that regardless of the differentiation time the Cell-IQ analysis software gave reliable neuronal cell counts with error of $\pm 10 \%$ (Supplemental Figure 3).

\section{Discussion}

Here, we showed that hESC-derived neuronal cells can be cultured and differentiated in an automated culture platform. Moreover, the automated culture and monitoring system enabled imaging of the entire hESC neuronal differentiation process using continuous timelapse recording. We also successfully used machine vision-based analysis software to automate the analysis of neuronal cultures.

The production of hESC-derived neuronal cells suitable for therapeutic interventions is currently under intense investigation. To achieve this goal, the culture procedures and characterization methods would benefit from any semiautomated or automated system that decreases the time-consuming laboratory work. Automated characterization methods would also make it easier to compare the results from different laboratories. Here, we tested a novel cell culture platform combined with an automated monitoring and analysis system, and showed that it is suitable for recording neuronal differentiation of hESCs. The system has also been successfully used to monitor and analyze the neural differentiation capacity of several hESC lines over time ${ }^{17}$ or human induced pluripotent stem cell lines (data not shown), thus there is no limitations on cell lines used. This platform has also been tested and found useful and reliable when analyzing the growth of other types of cells such as cardiomyocytes and hepatocytes (personal communication with Dr. Mari PekkanenMattila and Prof. Timo Otonkoski) and cytotoxicity. ${ }^{23}$ Because the culture plates were constantly in motion in the automated culture system, we plated hESC aggregates directly onto modified culture plates, similar to that described by Nat and co-workers, ${ }^{16}$ without first growing them as embryoid bodies. ${ }^{4,24}$ This allowed a constant recording of the entire neuronal differentiation process starting $2 \mathrm{~d}$ after induction. Monitored areas of interest were available for visual evaluation as images or movies. After the induction of differentiation, neuronal rosettes formed in the presence of FGF, as described previously $y^{4,24,25}$ Thereafter, neuronal cells grew rapidly from the mechanically separated and replated neuronal rosettes in the absence of FGF. In contrast to previous work using enzymatic treatments, ${ }^{4,26}$ here we used only mechanical dissection of the cultures. Although the rosette centers, neuronal centers, and cells survived without any growth factors for weeks, as previously reported, ${ }^{27}$ we added low concentrations of bFGF and BDNF to the medium to support neuronal proliferation (Supplemental online video 4) and maturation.

The automated culture platform described here offers a system in which a considerable number of cells can be monitored simultaneously (2- to 96- well plates in duplicate) for long periods. In our experiments we typically used 12- or 24 - well plates and monitored $\sim 100$ $\times(506 \times 675 \mu \mathrm{m})$ areas per plate (sizing from $1 \times 1$ to $6 \times 6$ grids). With these parameters, the monitoring interval for a particular area was $\sim 30 \mathrm{~min}$. This system proved to be particularly useful for monitoring the differentiation of neuronal cells, which form complex fine morphologic structures. We could also detect the non-neuronal population, defined earlier as flat cells, which existed in the differentiating cell population., ${ }^{2,428}$ These flat epithelial-like cells were immunonegative for neuronal and glial cell markers, consistent with an earlier report, ${ }^{4}$ partly immunopositive for nestin and A2B5 (data not shown) as described earlier, ${ }^{2}$ but mostly positive for a endothelial/fibroblast cell marker endoglin, that is, CD105. To our knowledge, this is the first article describing the phenotype of these flat epithelial-like cells. We detected a slow change of some of the flat cells into glial cells. This is consistent with earlier immunocytochemical analysis, ${ }^{2}$ showing that a portion of A2B5-positive and neuronal marker-negative cells in differentiating cultures give rise to GFAP-positive astrocytes. More importantly, the rapidly proliferating, flat epithelial-like cells overtake the cultures if they are not mechanically separated from the neuronal cultures or sorted out with negative selection using flow cytometry. ${ }^{29}$

Fiber growth and arborization are important for neuronal cell function. Neuronal fibers and cell numbers were quantified from the hESCderived neuronal populations using automated analysis software. The software provided rapid analysis without user intervention, in contrast to currently available protocols. ${ }^{11}$ The analysis was performed on the all-in-focus images of the living cells without the use of the labels, which enabled long-term studies of neuronal populations. Our results showed that if the neuronal population grew as a monolayer, the neuronal cell recognition protocol gave reliable cell counts when compared with FACS results and manual cell counting also regardless of the differentiation time. The density of the culture sets, however, limitations to the built neuronal cell recognition protocol which was designed to detect individual neurons from the cultures. Thus, we found that $\sim 100000$ proliferating neuronal cells $/ \mathrm{cm}^{2}$ can be imaged and analyzed for 1 week before the culture grows to confluent. For more confluent neuronal cultures, spe- cific recognition protocols based on area analysis can be developed similarly like for the growing hESC colonies. ${ }^{12}$ Further, in contrast to manual analysis, automatic analysis enabled thousands of cells to be counted and therefore the amount of data obtained was markedly increased and not biased due to the avoidance of human intervention. In addition, the FACS analysis demands samples with significantly higher number of cells which are not often easy to obtain. Classification of the glial cells using the cell recognition protocol we developed, however, was not reliable. This is most likely due to the rapid movement of the glial cells, which alters greatly their morphology from point to point. Thus, more detailed analysis protocols for reliable identification of glial cells need to be developed. Taken together, the results of the present study indicate that the morphologic classification of neuronal and glial cells from cultures in snapshot situations (e.g., quick microscopic evaluation of cell populations in culture, or evaluation of a single picture) can give rise to a false cell classification. Hence, immunocytochemical characterization is needed to confirm the morphologic evaluation if a continuous follow-up system is not available.

Other semi-automated culture systems have been developed that allow for mechanical passaging of hESC cultures ${ }^{6}$ or automated cell plating, media change, growth factor addition, and cell harvesting. ${ }^{7}$ Culture of hESCs and their derivatives also requires visual monitoring of colony growth and differentiation. The culture platform used in this study provides a stable atmosphere for the hESCs ${ }^{12}$ and neuronal cells, in which they can be automatically monitored and further analyzed in any point of the experiment. Moreover, e.g. this system provides tools for developing cell recognition protocols that can be used for optimizing differentiation procedures or as part of an automated quality control system.

\section{Conclusions}

These findings demonstrated that the use of an automatic cell culturing, monitoring, and analysis platform enables follow-up of the entire differentiation process from hESCs to mature neurons. This automatic system decreases the amount of labor and time required for cell culturing and analysis. Also, as the cells are kept in sterile conditions during the monitoring, they can be kept in prolonged culturing or to be used in following experiments such as transplantation studies. There are, however, some features such as user defined sample size that would facilitate analysis of different cell types according to their size as well as possibility to built up and 
modify cell recognition protocols by users themselves which would enhance the usage of the automated analysis software more widely. Finally, these types of automated monitoring and analysis processes can be used to develop enhanced cell differentiation procedures or as part of an automated quality control system for existing protocols.

\section{References}

1. Zhang SC. Neural subtype specification from embryonic stem cells. Brain Pathol 2006;16:132-42.

2. Carpenter MK, Inokuma MS, Denham J, et al. Enrichment of neurons and neural precursors from human embryonic stem cells. Exp Neurol 2001;172:383-97.

3. Reubinoff BE, Itsykson P, Turetsky T, et al. Neural progenitors from human embryonic stem cells. Nat Biotechnol 2001;19:113440.

4. Zhang SC, Wernig M, Duncan ID, et al. In vitro differentiation of transplantable neural precursors from human embryonic stem cells. Nat Biotechnol 2001;19:112933 .

5. Heikkilä TJ, Ylä-Outinen L, Tanskanen JM, et al. Human embryonic stem cell-derived neuronal cells form spontaneously active neuronal networks in vitro. Exp Neurol 2009;218:109-16.

6. Joannides A, Fiore-Hériché C, Westmore $\mathrm{K}$, et al. Automated mechanical passaging: a novel and efficient method for human embryonic stem cell expansion. Stem Cells 2006;24:230-5.

7. Terstegge S, Laufenberg I, Pochert J, et al. Automated maintenance of embryonic stem cell cultures. Biotechnol Bioeng 2007;96:195-201.

8. Conti L, Pollard SM, Gorba T, et al. Nicheindependent symmetrical self-renewal of a mammalian tissue stem cell. PLoS Biol 2005;3:e283.

9. Fico A, Manganelli G, Simeone M, et al. High-throughput screening-compatible single-step protocol to differentiate embryonic stem cells in neurons. Stem Cells Dev
2008;17:573-84.

10. Ying QL, Stavridis M, Griffiths D, Li M, Smith A. Conversion of embryonic stem cells into neuroectodermal precursors in adherent monoculture. Nat Biotechnol 2003;21:183-6.

11. Keenan TM, Hooker A, Spilker ME, et al. Automated identification of axonal growth cones in time-lapse image sequences. $\mathrm{J}$ Neurosci Methods 2006;151:232-8.

12. Narkilahti S, Rajala K, Pihlajamäki H, et al. Monitoring and analysis of dynamic growth of human embryonic stem cells: comparison of automated instrumentation and conventional culturing methods. Biomed Eng Online 2007;6:11.

13. Hovatta O, Mikkola M, Gertow K, et al. A culture system using human foreskin fibroblasts as feeder cells allows production of human embryonic stem cells. Human Reprod 2003;18:1404-9.

14. Inzunza J, Gertow K, Strömberg MA, et al. Derivation of human embryonic stem cell lines in serum replacement medium using postnatal human fibroblasts as feeder cells. Stem Cells 2005;23:544-9.

15. Rajala K, Hakala H, Panula S, et al. Testing of nine different xeno-free culture media for human embryonic stem cell cultures. Human Reprod 2007;22:1231-8.

16. Nat R, Nilbratt $M$, Narkilahti $S$, et al. Neurogenic neuroepithelial and radial glial cells generated from six human embryonic stem cell lines in serum-free suspension and adherent cultures. Glia 2007;55:385-99.

17. Lappalainen RS, Salomäki M, Ylä-Outinen L, et al. Similarly derived and cultured hESC lines show variation in their developmental potential towards neuronal cells in long-term culture. Regen Med 2010;5:749-62.

18. Lappalainen RS, Narkilahti S, Huhtala T, et al. The SPECT imaging shows the accumulation of neural progenitor cells into internal organs after systemic administration in middle cerebral artery occlusion rats. Neurosci Lett 2008;440:246-50.

19. Sonka M, Hlavac V, Boyle R. Image Processing, Analysis, and Machine Vision. 2nd edition. Pacific Grove, CA, USA:
Brooks/Cole Publishing; 1999.

20. Chapter 12: Object Recognition. In: Gonzalez R, Woods R. Digital Image Processing. Reading, MA, USA: Addison Wesley; 1993. pp. 861-902.

21. Tarvainen J, Saarinen M, Laitinen J, et al. Creating images with high data contents for microworld applications. Industrial Systems Review 2002:17-23.

22. Auer S, Lappalainen RS, Skottman H, et al. An antibody surface for selective neuronal cell attachment. J Neurosci Methods 2010; 186:72-6.

23. Toimela T, Tähti H, Ylikomi T. Comparison of an automated pattern analysis machine vision time-lapse system with traditional endpoint measurements in the analysis of cell growth and cytotoxicity. Altern Lab Anim 2008;36:313-25.

24. Li XJ, Du ZW, Zarnowska ED, et al. Specification of motoneurons from human embryonic stem cells. Nat Biotechnol 2005;23:215-21.

25. Benzing C, Segschneider M, Leinhaas A, et al. Neural conversion of human embryonic stem cell colonies in the presence of fibroblast growth factor-2. Neuroreport 2006;17:1675-81.

26. Schuldiner M, Eiges R, Eden A, et al. Induced neuronal differentiation of human embryonic stem cells. Brain Res 2001;913:201-5.

27. Joannides AJ, Fiore-Hériché $\mathrm{C}$, Battersby AA, et al. A scaleable and defined system for generating neural stem cells from human embryonic stem cells. Stem Cells 2007;25:731-7.

28. Gerrard L, Rodgers L, Cui W. Differentiation of human embryonic stem cells to neural lineages in adherent culture by blocking bone morphogenetic protein signaling. Stem Cells 2005;23:1234-41.

29. Sundberg M, Jansson L, Ketolainen J, et al. CD marker expression profiles of human embryonic stem cells and their neural derivatives, determined using flow-cytometric analysis, reveal a novel CD marker for exclusion of pluripotent stem cells. Stem Cell Res 2009;2:113-24. 